\title{
Utilização do PBL para o acompanhamento na aprendizagem de SCRUM
}

\author{
Carlos Henrique B. de Macedo ${ }^{1}$, Sarah Neves da S. Sotero ${ }^{1}$ \\ Danieli Silva de S. Rabelo ${ }^{1}$, Ricardo Alexsandro de M. Valentim ${ }^{1}$ \\ ${ }^{1}$ Laboratório de Inovação Tecnológica em Saúde (LAIS) - Universidade Federal do Rio \\ Grande do Norte (UFRN) - Natal, RN - Brazil \\ carlos.macedo@lais.huol.ufrn.br, sarah-neves2009@hotmail.com, \\ rabrlodanni@gmail.com, ricardo.valentim@ufrnet.br

\begin{abstract}
This study reports on the efficiency of learning processes, based on the Problem Based Learning (PBL) methodology applied to Scrum, which is used in complex software project management and planning and differs from the system. traditional management. Both adopted by the School of Programming (SP) of the Laboratory of Technological Innovation in Health (LAIS), deepening in how PBL linked to Scrum learning can be effective in increasing student performance.
\end{abstract}

Keywords: PBL. Scrum. Methodology Efficient.

Resumo. Este estudo relata a eficiência de processos de aprendizagem, tendo como pilar a metodologia Problem Based Learning(PBL), ou Aprendizado Baseado em Problemas (ABP) aplicada ao Scrum, que é utilizada em gestão e planejamento de projetos de softwares complexos e difere do sistema tradicional de gerenciamento. Ambos adotados pela Escola de Programação(EP) do Laboratório de Inovação Tecnológica em Saúde(LAIS), aprofundando-se em como o PBL atrelado à aprendizagem de Scrum pode ser eficiente em aumentar o desempenho dos alunos.

Palavras-chave: PBL. Scrum. Metodologia Eficiente.

\section{Introdução}

Atualmente, uma grande parte das instituições ainda fazem uso do método tradicional de ensino baseado na divisão de disciplinas, onde o professor é o detentor de todo conhecimento e o aluno apenas recebe as informações. Essa forma de aprendizado é desgastante para o aluno, tornando a rotina de sala de aula cansativa, e um baixo rendimento do discente, pois grande parte das informações não são absorvidas[1].

Nesse contexto, surgiu a necessidade em buscar outras alternativas de ensino que tivesse como foco o aluno. Pensando nisso, no final da década de 60 surgiu o PBL na Faculdade de Medicina da Universidade McMaster em Hamilton, Canadá [3].

O PBL tem como foco o aluno, o intuito é fazer uso de problemas para que possam buscar, e aplicar seus conhecimentos para a resolução dos mesmos. Dessa forma, fazendo-os desenvolver habilidades de pensamento crítico e de resolução ágil. Seus resultados são tão eficientes que várias instituições têm aderido. 
Passamos por constantes mudanças, e hoje vivenciamos a era digital, da computação, IA (Inteligência Artificial), e muitas outras tecnologias. De fato a era da informação traz grandes mudanças, e precisamos acompanhá-las. Dessa forma entra em cena a ferramenta Scrum, um framework de gerenciamento de projetos, mais conhecida no campo da tecnologia da informação. Normalmente é utilizada para gestão em projetos de software em grupos, trazendo mais eficiência na interação da equipe e no desenvolvimento dos projetos efetivando um bom resultado do produto final. O Scrum é um módulo de aprendizagem exigido entre os componentes curriculares do aluno na Escola de Programação do LAIS.

Seguidamente, será especificado como essas duas ferramentas se caracterizam, e como o PBL potencializa ainda mais a efetividade do aprendizado do Scrum, apresentando seus resultados aplicados na rotina dos alunos da EP.

\section{Desenvolvimento}

\subsection{Como se caracteriza o PBL}

A prática da metodologia de aprendizagem PBL gira sempre em torno de um determinado problema e é desenvolvida paralelamente à estratégia da sala de aula invertida, onde os alunos irão adquirir conhecimentos por meio de pesquisas, seja em bibliotecas, internet ou em outros meios de informação, deixando de lado os paradigmas tradicionais do ensino presencial, obtendo as soluções desejadas. As imagens a seguir mostram o fluxo organizacional da metodologia, avaliação e execução.

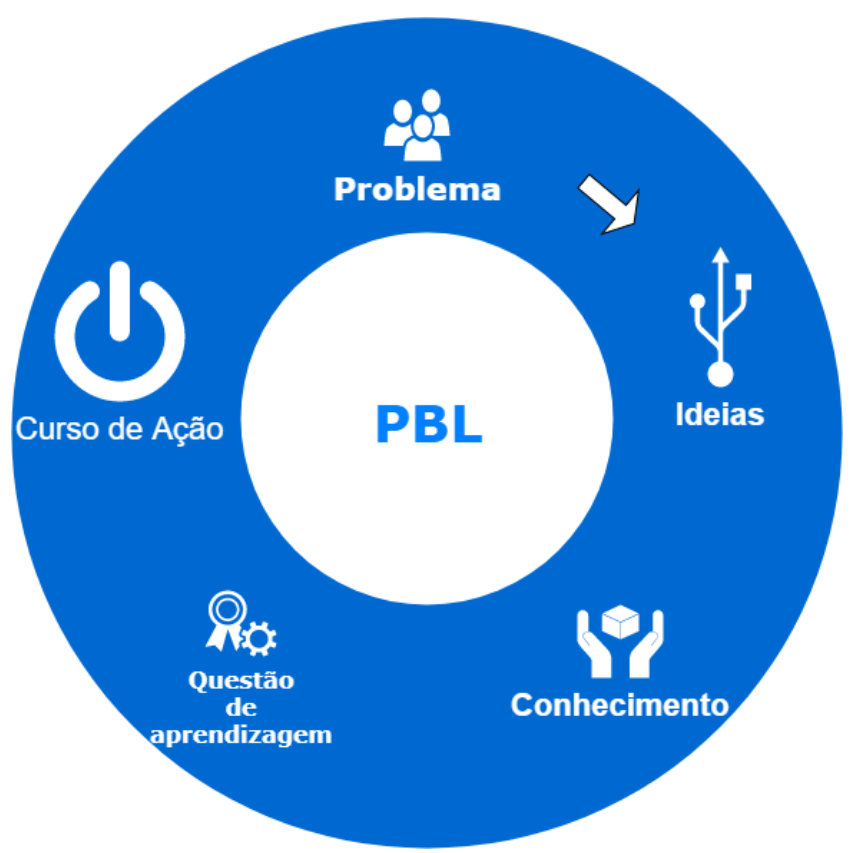

Figura 1

Fonte: Os Autores. 


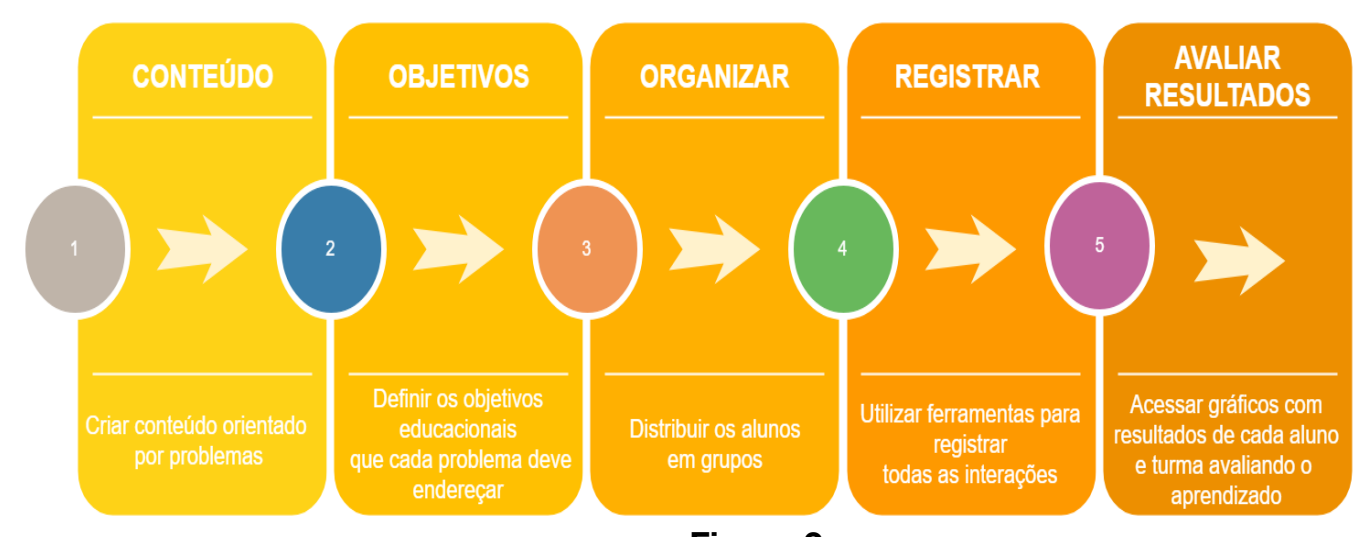

Figura 2

Fonte: Os Autores.

\subsection{Como se caracteriza o SCRUM}

O Scrum, como já citado, é uma ferramenta de gerenciamento de projetos de software, nele os projetos são separados em ciclos, os quais representam um time-box onde um prazo é definido para a realização das atividades, e esses ciclos são chamados de sprints. Existe um time Scrum que é formado por um PO (Product Owner), Scrum Master e Time de Desenvolvimento[2]. O PO é responsável por definir o backlog do produto e garantir que fique claro para a equipe o que é necessário. O Scrum Master é um facilitador, seu papel é conduzir a equipe de desenvolvimento e remover quaisquer obstáculos encontrados pelos mesmos. O time de desenvolvimento é responsável por desenvolver o produto atendendo ao que foi solicitado pelo PO.

Vários outros eventos transcorrem no Scrum, são eles: Reunião de Planejamento da Sprint; Reunião Diária; Revisão do Sprint; Retrospectiva da Sprint; logo o acompanhamento da aprendizagem dos alunos é fundamental para o desenvolvimento das suas habilidades[2].

Todos esses elementos citados fazem parte da ementa na EP, e são conteúdos que precisam ser aprendidos pelos alunos ao cursar o módulo.

\subsection{Aplicação do PBL na EP voltado ao aprendizado de SCRUM.}

Na Escola de Programação, é necessário que os alunos obtenham o Certificado de Fundamentos Básicos Scrum, com o intuito de adquirir domínio da ferramenta na execução dos projetos da escola. Para obter essa certificação é necessário estudar todo um material extenso, com muitos conceitos sobre o assunto para a realização da prova.

No decorrer das aulas dos princípios do Scrum a metodologia PBL é aplicada de modo a sair do conceito teórico e promover uma maior interação entre os alunos com a ferramenta, pondo em prática os conceitos do Scrum, tornando o aprendizado mais flexível e proveitoso. Frequentemente, são aplicados PBL Forms (Formulários PBL) no qual os alunos terão que listar os termos difíceis, objetivos de aprendizado, sistematização de sugestões, entre outras tarefas com base no problema que foi apresentado. O PBL forms busca guiar o aluno no processo de aprendizagem, tornando o desafio proposto mais claro, facilitando sua visualização e agilizando sua resolução.

Quando atrelado o PBL ao estudo do Scrum, as chances de obtenção de certificação aumentam drasticamente, pois como a metodologia usa o aprendizado do 
VIII Congresso Brasileiro de Informática na Educação (CBIE 2019)

Anais do XXV Workshop de Informática na Escola (WIE 2019)

Scrum aplicado a problemas, os alunos absorvem o conhecimento adquirido na prática com maior eficiência.

\section{Resultados e discussões}

O processo para a obtenção da certificação teve início aplicando a sala de aula invertida, e utilizamos um grupo composto por 12 alunos onde o grupo teve que estudar o material fornecido e buscar conhecimentos fora do ambiente escolar. Em seguida, foram ministradas aulas por um por um Scrum Master com a finalidade de sanar dúvidas e fazer a imersão em conceitos relacionados à metodologia de interesse. A partir disso, foi possível seguir para a prática implementando jogos, simulados Scrum. Além disso, os projetos do laboratório como GEMA, Autonomus, HERA, Hemodinâmica, entre outros, fazem uso de Scrum em seu desenvolvimento, dos quais os alunos participavam.

Os passos descritos anteriormente referem-se às etapas do PBL, como elas foram elencadas na potencialização do aprendizado para obter a certificação. Dessa forma, observou-se um grande percentual de alunos aprovados na primeira tentativa da prova de Certificado de Fundamentos Básicos Scrum.

Um questionário também foi aplicado aos alunos da Escola de Programação, propondo que os mesmos avaliassem a significância do PBL no aprendizado de Scrum. Os resultados evidenciaram a eficácia de sua aplicação no aprendizado do framework citado, mostrando como o PBL foi importante para a formação dos alunos da EP.

\section{Qual a importância do PBL pra o aprendizado de SCRUM?}

\section{2 respostas}

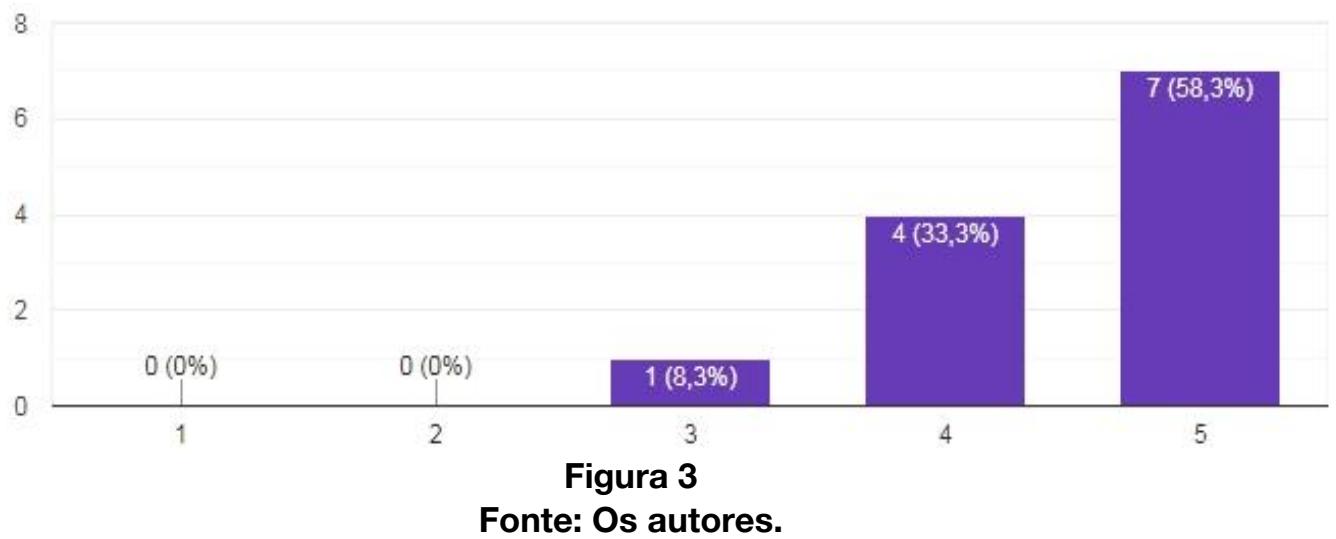


VIII Congresso Brasileiro de Informática na Educação (CBIE 2019)

Anais do XXV Workshop de Informática na Escola (WIE 2019)

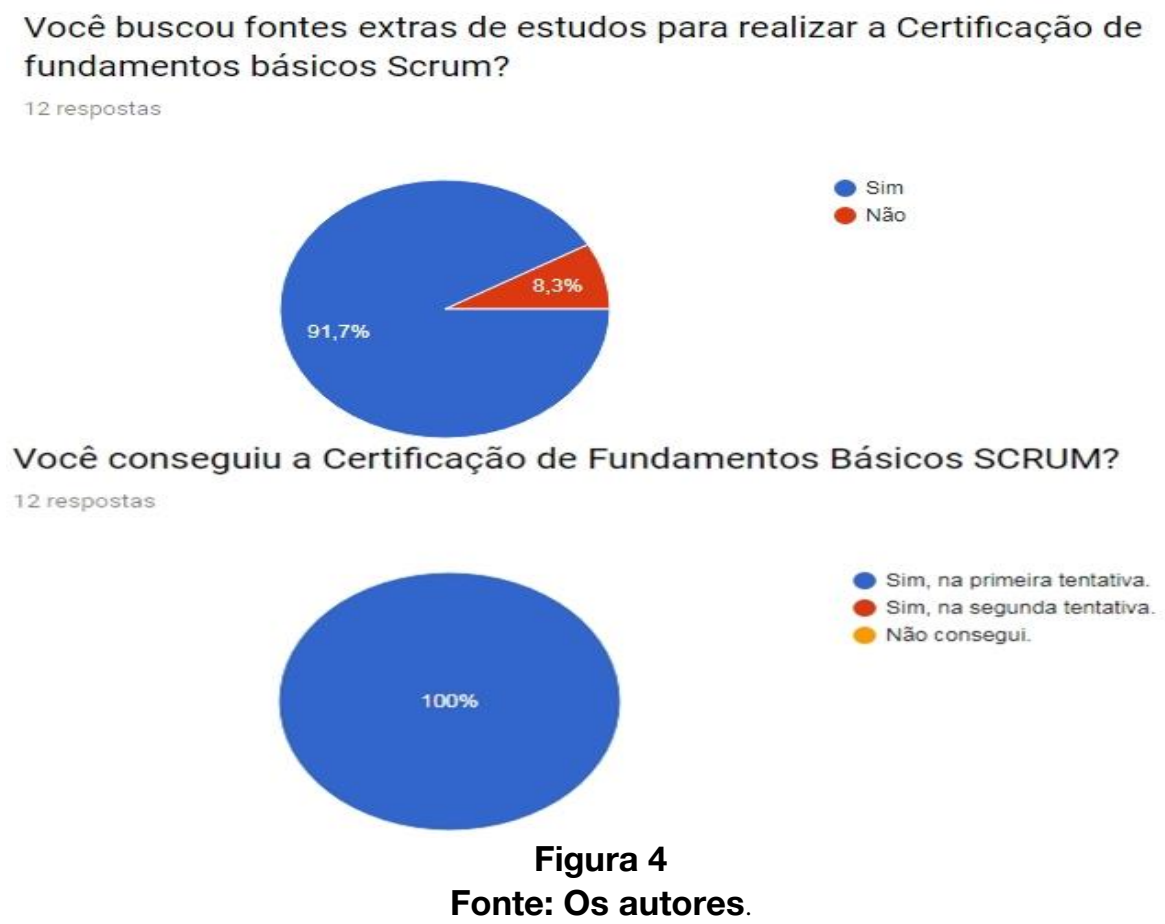

\section{Conclusão}

Logo, com os resultados positivos após a aplicação dessa metodologia no aprendizado de Scrum, considerou-se que o PBL é uma ferramenta importante no processo de aprendizagem dos estudantes, e também pode ser utilizada integralmente a outros frameworks e métodos buscando acompanhar e otimizar o aprendizado dos alunos.

\section{Referências bibliográficas}

[1] SOUZA, Denise; VERGOTTINI, Viviane; BERNINI, Denise Simões Dupont. Educação dos tempos modernos através da aprendizagem colaborativa: uma abordagem sobre EDUSCRUM. Anais do Xxix Simpósio Brasileiro de Informática na Educação (sbie 2018), [s.1.], p.51-60, 28 out. 2018. Brazilian Computer Society (Sociedade Brasileira de Computação - SBC). http://dx.doi.org/10.5753/cbie.sbie.2018.51.

[2] CARVALHO, Bernardo Vasconcelos de; MELLO, Carlos Henrique Pereira. Aplicação do método ágil scrum no desenvolvimento de produtos de software em uma pequena empresa de base tecnológica. Gest. Prod., São Carlos, V. 19, N. 3, P. 557-573, 2012, Itajubá, Mg, Brasil, v. 19, n. 3, p.557-573, 20 abr. 2012.

[3] http://lagarto.ufs.br/uploads/content_attach/path/11327/metodologia_da_abp_0.pdf . 